\title{
A probiotic and mixed-enzymes combination reduces the inflammatory response, faecal shedding and systemic spread of Campylobacter jejuni in broilers
}

\author{
K. Gibbs ${ }^{1 *}$, L. Lacharme-Lora ${ }^{2}$, Y. Dersjant-Li ${ }^{1}$, C. Evans ${ }^{1}$ and P. Wigley ${ }^{2}$ \\ ${ }^{1}$ Danisco Animal Nutrition, Willem Einthovenstraat 4, 2342 BH Oegstgeest, the Netherlands; ${ }^{2}$ Institute of Infection E Global \\ Health, University of Liverpool, Chester high road, Neston, CH64 7TE, United Kingdom; kirsty.gibbs@iff.com
}

Received: 11 February 2021 / Accepted: 13 April 2021

(c) 2021 K. Gibbs et al.

\section{RESEARCH ARTICLE}

POULTRY

\begin{abstract}
Recent research has questioned the notion that Campylobacter jejuni is a harmless resident of the avian gastrointestinal tract (GIT). The following trial examined the effect of dietary supplementation with a mixture of enzymes and Bacillus-based probiotics on growth performance, C. jejuni colonisation, GIT immune responses, faecal shedding and extra-intestinal spread in broilers. Fifty-eight, Ross $308 \mathrm{~d}$-old broilers were randomly assigned to one of four treatments, giving 14 or 15 birds/pen. Birds were given nutritionally complete, complex, phased diets unsupplemented (two treatments) or supplemented (two treatments) with a multi-enzyme containing 2,000 U/kg xylanase, $200 \mathrm{U} / \mathrm{kg}$ amylase and 4,000 U/kg protease, and 75,000 cfu/g of a combination of three strains of Bacillus amyloliquefaciens. One control and one supplemented diet group contained birds orally challenged with $10^{5} \mathrm{cfu} C$. jejuni strain M1 on $\mathrm{d} 21$ of age. Among challenged birds, the mixed-enzymes and probiotic combination numerically reduced faecal $C$. jejuni shedding (-98\% vs challenged control) three days-post-infection (d.p.i.), and at 7 d.p.i. numerically reduced C. jejuni colonisation of the ileal mucosa (-1000-fold vs control) and totally inhibited systemic spread of $C$. jejuni to the liver, compared to the control $(P<0.05)$. It suppressed early pro-inflammatory chemokine response seen in the ileum, caecum and caecal tonsil tissues (at 3 d.p.i.) in challenged control birds $(-18$ to -46 -fold; $P<0.05)$ and altered expression of pro-inflammatory (IL-1 $\beta$, IL-6, IL-17A and IL-17F) and regulatory (IL-10 and TGF- $\beta$ ) cytokines. The data demonstrated inhibition of a sustained pro-inflammatory response to $C$. jejuni infection and improved intestinal barrier integrity in supplemented birds. This highlighted the importance of looking beyond simple measurements of feed conversion and body weight gain when seeking to understand the effects and mode of action of poultry dietary interventions. Achieving a favourable balance between the gut, immune function, microbiome and nutrition should be the goal for achieving good gastrointestinal health and optimal performance.
\end{abstract}

Keywords: Bacillus amyloliquefaciens, Campylobacter jejuni, enzymes, immunity, gut health, poultry

\section{Introduction}

Campylobacter jejuni is a Gram-negative, spiral rod-shaped bacterium that is pathogenic to humans. It is the leading cause of human gastroenteritis globally, with 246,307 confirmed campylobacteriosis cases reported across the EU in 2016, and the incidence is increasing (EFSA, 2017). Poultry meat is the major source of infection. An estimated
$75 \%$ of broiler carcasses are contaminated with C. jejuni across the EU (EFSA, 2011), representing a substantial risk to public health due to the handling and consumption of contaminated meat and meat products. On-farm strategies aimed at reducing Campylobacter spp. incidence and spread before slaughter and carcass handling are, therefore, of considerable interest. Alongside this, the worldwide prohibition and/or phasing out of antibiotics as growth 
promoters, due to consumer pressure and concerns about the spread of human-antibiotic-resistance, is fuelling a need for alternative solutions that support gut health and immunity in broiler production.

Such strategies are attractive from both a human health and broiler production point of view. C. jejuni has traditionally been considered a commensal in poultry, typically colonising the avian gastrointestinal tract (GIT), mainly the caeca, colon and cloaca, at high levels ( $9 \log _{10}$ cfu/g) without any apparent pathology (Dhillon et al., 2006; Shane, 1992). However, emerging evidence has questioned this notion of commensalism in poultry, as in vitro and in vivo studies have observed an innate inflammatory response soon after a C. jejuni infection This is characterised by increased production of pro-inflammatory cytokines (Smith et al., 2005, 2008), early increases in circulating monocytes/macrophages (Meade et al., 2009) and the activation of pattern recognition receptors, including toll-like receptors (TLRs) that are a key part of the innate immune response (De Zoete et al., 2010). Whilst these responses are often transient and without accompanying clinical symptoms, recent studies reported that infection can lead to a prolonged inflammatory response, immune dysregulation and clinical disease symptoms, including caecal inflammation, diarrhoea and pododermatitis. This may be linked to host genetics (Humphrey et al., 2014) and timing of exposure (Connerton et al., 2018). C. jejuni colonisation can alter the gut microbiota (Kaakoush et al., 2014) which may reduce feed conversion and impair growth performance (Stanley et al., 2016). Any interaction between host and bacteria that diverts resources and energy away from maintenance and growth is undesirable in a production setting. Novel strategies for supporting the optimal functioning of the broiler GIT through positive and efficient interactions between the gut and immune function, microbiome and nutrition in the presence of $C$. jejuni are therefore desirable.

Exogenous feed enzymes and probiotics (known as directfed microbials) are increasingly being used in poultry production to achieve this positive and efficient interaction, with the aim of supporting gut health and improving growth performance. There is current interest in their use as part of a suite of strategies to replace the need for antibiotics. In broilers, probiotics can improve growth performance and feed digestibility (Dersjant-Li et al., 2014; FAO, 2016; Wealleans et al., 2017), exert beneficial immunomodulatory effects in the GIT (Lee et al., 2010; Waititu et al., 2014), improve gut epithelial integrity (Gadde et al., 2017) and beneficially alter the gut microbiota (La Ragione and Woodward, 2003; Park and Kim, 2014). They are frequently used in combination with exogenous enzymes, such as fibredegrading xylanase, starch digesting amylase and protein hydrolysing proteases. Such combinations can produce greater improvements in nutrient digestibility than when delivered individually (Olukosi et al., 2015; Romero et al., 2013, 2014; Wealleans et al., 2017).

A unique mix of enzymes and a three-strain Bacillus-based probiotic combination has proven effective at improving poultry growth performance, nutrient and energy digestibility and animal welfare parameters in both research and commercial settings (Dersjant-Li et al., 2015; Flores et al., 2016; Wealleans et al., 2017). However, its impact on the infection biology of industry relevant microbes is unclear. The aim of this study was to determine the effect of a mixedenzyme and Bacillus-based probiotic combination on $C$. jejuni colonisation, gut immune responses, faecal shedding and extra-intestinal spread in broilers orally challenged with a clinically relevant strain of $C$. jejuni.

\section{Materials and methods}

All animal work was conducted under accordance with UK legislation, Animals (Scientific Procedures) Act 1986, under authority of Project License. All work was approved by the University of Liverpool Animal Welfare and Ethical Review Body prior to the award of the license. All birds were checked at least twice daily to ensure their health and welfare was maintained.

\section{Exogenous enzymes and probiotic}

A commercial mixed-enzymes and probiotic (MEP) preparation was utilised (Syncra ${ }^{\circledR}$ AVI, Danisco Animal Nutrition, Marlborough, UK) containing a combination of endo-1,4- $\beta$-xylanase (EC 3.2.1.8) originating from Trichoderma reesei, $\alpha$-amylase (EC 3.2.1.1) originating from Bacillus licheniformis and serine protease (EC 3.4.21.62) originating from Bacillus subtilis, providing 2,000 U/kg feed xylanase, $200 \mathrm{U} / \mathrm{kg}$ amylase and 4,000 U/kg protease. The product contained a combination of three strains of Bacillus amyloliquefaciens, formulated to deliver $75,000 \mathrm{cfu} / \mathrm{g}$ feed. Internally validated methods were used to confirm additive inclusion in final feed samples, using xylanase activity as a marker of enzyme recovery. These analyses were conducted by Danisco Animal Nutrition Innovation Laboratories (Brabrand, Denmark). The presence of the probiotic was confirmed by a total Bacillus spp. enumeration count and identification of the three specific strains by colony morphology.

\section{Experimental and control diets}

A basal control diet containing corn, soybean meal, wheat, barley and rapeseed meal and added vitamins and minerals, was formulated to meet the recommended nutritional requirements of the birds during starter (days 0 to 10) and grower (days 11 to 28) phases (NRC, 1994), except for a moderate down specification of energy (of $0.41 \mathrm{MJ}$ $\mathrm{ME} / \mathrm{kg}$ feed) in line with recommended nutritional matrix 
values for the mixed-enzymes component (Table 1). All diets contained $500 \mathrm{FTU} / \mathrm{kg}$ of a commercial Buttiauxella spp. phytase (Axtra ${ }^{\circ}$ PHY, Danisco Animal nutrition, Marlborough, UK). The basal diet was supplemented with the MEP at the dose levels described above for the experimental treatments. Diets were provided to birds ad libitum in crumble (starter) or pellet (grower) form, from day 1 to 28 , and birds had free access to water.

\section{Birds, housing and experimental design}

One day old, Ross 308 broilers of mixed gender were obtained from a commercial hatchery where they had been vaccinated against infectious bronchitis. The birds were assigned to floor pens based on body weight (BW), so that pens contained approximately equal average bird weight. Pens were bedded with dry, clean wood shavings. A total of 58 birds were assigned to four treatments, giving 14 or 15 birds per pen with a single pen per treatment. The four treatments comprised: (1) unchallenged birds receiving the control diet ('unchallenged control'); (2) C. jejuni challenged birds receiving the control diet ('challenged control)'; (3) unchallenged birds receiving the control diet supplemented with the MEP; (4) C. jejuni challenged birds receiving the control diet supplemented with MEP. Pens were located in an environmentally controlled room in which temperature was maintained at $30^{\circ} \mathrm{C}$ until 3 weeks of age and then reduced to $20^{\circ} \mathrm{C}$ for the remainder of the study period. Three days prior to infection, all birds were confirmed to be Campylobacter negative by cloacal swabbing.

\section{Campylobacter jejuni challenge}

Birds in the challenged groups were orally gavaged on day 21 with $10^{5} \mathrm{cfu} C$. jejuni strain M1 (an experimental strain originally isolated from a human case of campylobacteriosis). The unchallenged birds received an oral inoculation of sterile saline as a placebo. On days 24 and 28 of age, seven or eight birds respectively per treatment group were euthanised by cervical dislocation and the contents of the ileum and caecum extracted by gentle mechanical pressure and flushing with distilled water for the analysis of C. jejuni intestinal load. From the ileal/ caecal digesta and the mucosa samples, $1 \mathrm{~g}$ was diluted in nine volumes of maximum recovery diluent (MRD) and then serial 10-fold dilutions (in MRD) were made, prior to plating, in triplicate, $20 \mu \mathrm{l}$ spots on to selective blood-free agar (mCCDA) plates (Lab M, Heywood, Lancashire, UK) supplemented with Campylobacter enrichment supplement (SV59; Mast Group, Bootle, Merseyside, UK) for counting of the number of colony forming units, in accordance with the Miles and Misra method (Miles et al., 1938).

Table 1. Ingredient and calculated nutrient composition ( $\mathrm{g} / \mathrm{kg}$, as fed) of the negative control basal diets given in the starter ( $\mathrm{d} \mathrm{0-10)} \mathrm{and}$ grower (d 11-28) phases. ${ }^{1}$

$\begin{array}{llcc} & & & \text { Finisher (days 11-28) } \\ \text { Ingredient (g/kg) } & \text { Corn } & \text { Starter (days 0-11) } & 302.8 \\ & \text { Soybean meal (48\% CP) } & 358.7 & 257.4 \\ & \text { Wheat } & 284.5 & 250 \\ & \text { Barley } & 200 & 70 \\ & \text { Rapeseed meal } & 50 & 50 \\ & \text { Soybean oil } & 50 & 9.5 \\ & \text { Limestone } & 14.7 & 10.2 \\ & \text { Dicalcium phosphate } & 11.4 & 35.2 \\ & \text { Vegetable fat blend } & 13.3 & 2.8 \\ & \text { L-Lysine } & & 2.7 \\ & \text { DL-methionine } & 4 & 1.0 \\ & \text { L-threonine } & 3.4 & 3.4 \\ & \text { NaCl } & 1.6 & 5 \\ & \text { Limestone } & 3.4 & 208 \\ & \text { Vitamin-mineral premix } & 11.4 & 29.2 \\ & \text { Crude protein (CP) } & 5 & 12.7 \\ & \text { Crude fibre } & 220 & 7.7 \\ & \text { Metabolisable energy (MJ/kg) } & 29.5 & 3.0 \\ & \text { Ca } & 12.2 & 1.7 \\ & \text { P (available) } & 9.2 & 11 \\ & \text { Na } & 3.5 & 8.3 \\ & \text { av. lysine } & 1.7 & 7.2 \\ \text { av. methionine+cysteine } & 12.5 & 2.1\end{array}$

${ }^{1}$ Both diets were top-dressed with $500 \mathrm{FTU} / \mathrm{kg}$ Axtra ${ }^{\circledR} \mathrm{PHY}$ (Danisco Animal Nutrition, Marlborough, UK).

2 Supplied per kilogram of diet: antioxidant (ethoxyquin), $100 \mathrm{mg}$ : biotin, $0.2 \mathrm{mg}$; calcium pantothenate, $12.8 \mathrm{mg}$; cholecalciferol, $60 \mu \mathrm{g}$; cyanocobalamin, $0.017 \mathrm{mg}$; folic acid, $5.2 \mathrm{mg}$; menadione, $4 \mathrm{mg}$; niacin $35 \mathrm{mg}$; pyridoxine, $10 \mathrm{mg}$; trans-retinol, $3.33 \mathrm{mg}$; riboflavin, $12 \mathrm{mg}$; thiamine, $3.0 \mathrm{mg}$; DL-a-tocopheryl acetate, $60 \mathrm{mg}$; choline chloride, 638 mg; Co, 0.3 mg; Cu, 3.0 mg; Fe, 25 mg; I, 1 mg; Mn, 125 mg; Mo, 0.5 mg; Se, 200 pg; Zn, 60 mg. 


\section{Faecal shedding}

Fresh faecal samples were collected on a per bird basis from bacterially-challenged birds on day 24 of age ( 3 d.p.i.) and day 28 (7 d.p.i.), for the quantification of $C$. jejuni, by the same method described above. To ensure faecal matter was fresh and to avoid litter contamination, five birds per treatment were placed into a box on clean paper for holding and faecal matter collected as soon as available.

\section{Extra-intestinal spread of Campylobacter jejuni}

Livers were removed from birds euthanised on day 24 and 28 of age and used for quantitative and qualitative analyses of systemic spread of the bacteria. Liver tissue $(\sim 1 \mathrm{~g})$ was homogenised in nine volumes of MRD and plated directly onto Campylobacter selective agar (Lab $\mathrm{M}$, Heywood, Lancashire, UK) for counting the number of colony forming units, as described above. Separate swabs of each liver homogenate were enriched in Exeter broth (Lab M, Heywood) before plating on to agar to confirm the number of livers testing negative or positive for $C$. jejuni.

\section{Intestinal cytokine and chemokine expression}

Samples ( $10 \mathrm{~cm}$ in length) of ileal, caecal and caecal tonsil tissues from birds euthanised at each time-point were collected and stored in $500 \mu \mathrm{l}$ RNA at $-20^{\circ} \mathrm{C}$ (Sigma, Poole, Dorset, UK). Total RNA was isolated from 20 to 30 mg tissue using the RNeasy minikit (Qiagen, West Sussex, UK) in accordance with the manufacturer's instructions. The isolated RNA was then eluted into $50 \mu \mathrm{l}$ RNase-free water and total RNA was determined using a NanoDrop (ND-1000) spectrophotometer. The expression of mRNA for chemokine CXCLi2 (chemokine [C-X-C motif] ligand i2] and cytokines interleukin-1 $\beta$ (IL-1ß), interleukin-6 (IL6), interleukin-17A (IL-17A), interleukin-17F (IL-17F), interleukin-10 (IL-10) and transforming growth factor-beta (TGF- $\beta 4$ ), were measured by quantitative real-time reverse transcription polymerase chain reaction (qRT-PCR), using procedures and equipment as described by Humphrey et al. (2014). Primer and probe sequencing was conducted as described by Shini and Kaiser (2009), as the reference gene used was 28S rRNA. Expression of the target genes was determined as being the difference between the cycle threshold $\left(\mathrm{C}_{\mathrm{T}}\right)$ of the target gene and that of the reference gene $\left(\Delta C_{T}\right)$. Results were presented as fold-changes in corrected target gene expression in $C$. jejuni challenged birds $\left(\Delta \mathrm{C}_{\mathrm{T}}\right)$ relative to that in control (unchallenged) birds $\left(2-\Delta \Delta \Delta \mathrm{C}_{\mathrm{T}}\right)$.

\section{Statistical analyses}

Data relating to microbial counts and immunological parameters were collected and analysed on an individual bird basis. The data were analysed by Wilcoxon-signed-rank tests to determine differences between the supplemented and control diet groups using JMP 11 version 11 (SAS, Institute Inc., Cary, NC, USA). Differences were considered significant at $P<0.05$. Where $0.05<P<0.1$, this was considered a tendency.

\section{Results}

\section{Diet analysis}

Analysed activities of xylanase in the additive supplemented diets were within $10 \%$ of target doses. Bacillus recovery was within $0.75 \mathrm{log} \mathrm{cfu} / \mathrm{g}$ of target dose. Bird growth performance was not assessed in this study due to insufficient pen replicates, however no obvious differences in growth between treatments were apparent.

\section{Campylobacter jejuni intestinal colonisation}

Unchallenged birds remained Campylobacter negative for the entire study length. Among challenged birds, $C$. jejuni counts (cfu/g) were obtained from at least one tissue sample (ileal/caecal lumen/mucosa) in all but one replicate at 3 d.p.i. and from all birds at 7 d.p.i. As expected, counts were generally higher (by several orders of magnitude) in caecal - compared with ileal-tissue and were higher at 7 d.p.i. compared with 3 d.p.i., regardless of dietary treatment (data not shown). There were no significant effects of MEP on gastrointestinal C. jejuni counts $\left(\log _{10} \mathrm{cfu} / \mathrm{g}\right)$ in challenged birds at 3 d.p.i. (data not shown). At 7 d.p.i., $C$. jejuni counts were numerically lower (by, on average, 1000fold) in the ileal mucosa of MEP supplemented challenged birds compared with control challenged birds $(P=0.331)$, but counts in the ileal lumen did not differ between groups.

\section{Faecal shedding}

Figure 1 shows the effects of the MEP on faecal shedding of C. jejuni following oral challenge, at 3 d.p.i. (Figure 1A) and 7 d.p.i. (Figure 1B). At 3 d.p.i., faecal counts were numerically reduced (by, on average, $98 \%$ or $1.63 \log$ ) in MEP supplemented challenged birds compared with challenged control birds, but this did not attain statistical significance $(P=0.424)$. No differences between treatments were evident at 7 d.p.i. $(P>0.05)$.

\section{Bacterial translocation}

At 3 d.p.i., there was no evidence of $C$. jejuni in the livers of MEP supplemented or control groups prior to challenge. After challenge, one out of eight liver samples was identified as positive for $C$. jejuni, from a MEP supplemented bird (data not shown). In contrast, at 7 d.p.i., C. jejuni was present in the livers of seven out of eight challenged control birds but was not present in MEP supplemented challenged birds (median $2.59 \log _{10} \mathrm{cfu} / \mathrm{g}$ vs $0.0 \mathrm{cfu} / \mathrm{g}$ or 

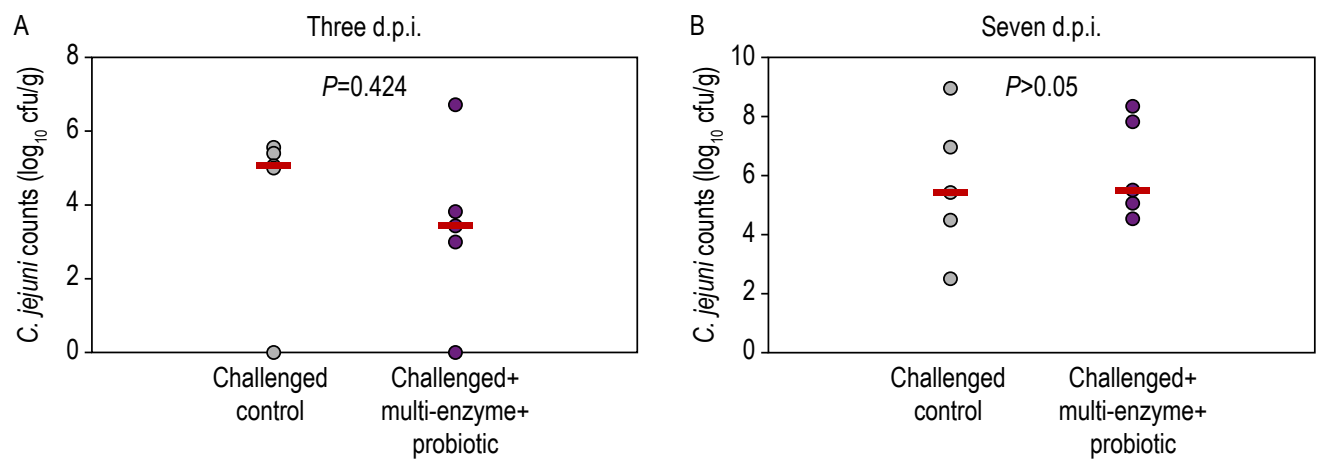

Figure 1. Faecal shedding of Campylobacter jejuni $\left(\log _{10} \mathrm{cfu} / \mathrm{g}\right)$ in broilers receiving diets with and without a mixed-enzymes and probiotic combination (A) 3 and (B) 7 days post-infection (d.p.i.) ( $n=5$ ). (Red bars represent median values).

undetectable by direct culture, respectively $(P<0.05$; Figure $2 \mathrm{~A})$. In total, $86 \%$ of livers from the control challenged group were positive for $C$. jejuni, whilst none of the livers from the additive supplemented group were (Figure 2B).

\section{Chemokine and cytokine expression}

The median relative expression of proinflammatory chemokine CXCLi2 in gastrointestinal tissue from $C$. jejuni challenged birds with and without MEP supplementation is presented in Figure $3(\mathrm{~A}-\mathrm{F})$. There was a clear spike in CXCLi2 expression throughout the GIT at 3 d.p.i. in the challenged control birds, which was absent or markedly reduced in MEP supplemented challenged birds. The average relative CXCLi2 expression was 18-, 22- and 46fold higher, respectively, in ileal, caecal and caecal tonsil tissues from the control vs MEP supplemented birds at 3 d.p.i. $(P<0.01$ in all cases). By 7 d.p.i., CXCLi2 expression was moderate in both treatment groups, and did not differ in ileal or caecal tonsil tissues but was higher in the caeca of challenged control birds than in MEP supplemented birds (relative expression 5.87 vs $0.26 ; P<0.01$; Figure $3 E$ ).

Relative expression of pro-inflammatory cytokines IL-1 $\beta$, IL-6, IL-17A and IL17F, and regulatory cytokines IL-10 and TGF- $\beta 4$ in $C$. jejuni challenged birds, is presented in Table 2. Three days after oral C. jejuni challenge, birds supplemented with MEP demonstrated elevated levels of IL-1 $\beta$ in caecal tissue and caecal tonsils compared with challenged control birds ( $P<0.01$ and $P=0.109$ respectively), but by 7 d.p.i. expression levels were reduced (Table 2). In contrast, in the ileum, IL-1 $\beta$ expression was similar between groups at 3 d.p.i. but rose markedly in the challenged control group at 7 d.p.i. $(P<0.05$, Table 2$)$. Effects on IL-17A and IL-6 expression followed a similar trend, being higher in the challenged supplemented birds at 3 d.p.i., but lower in supplemented challenged birds at 7.d.p.i. vs challenged control birds. Expression of IL17F showed no differences
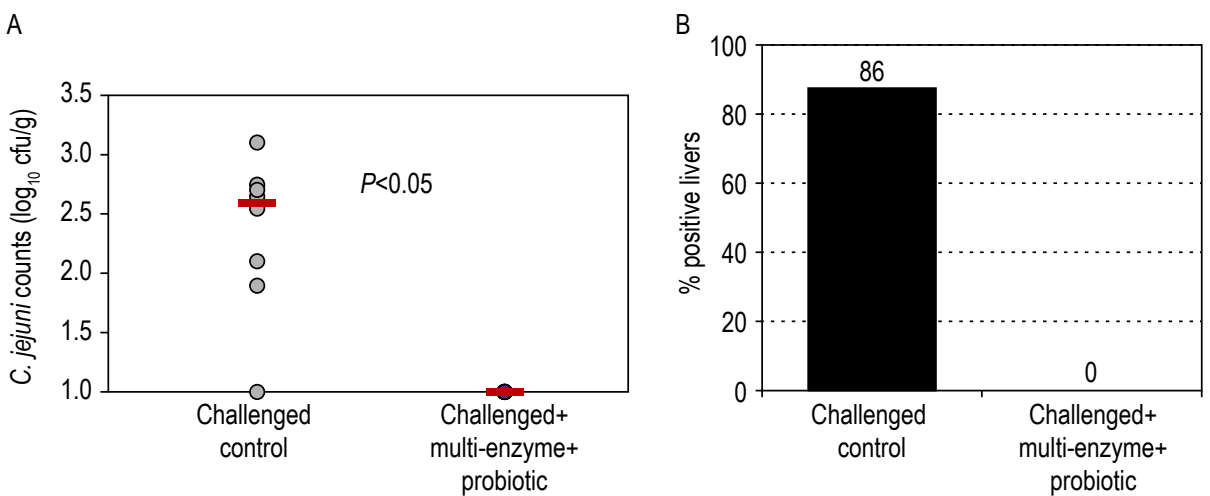

Figure 2. Extraintestinal spread of Campylobacter jejuni in broilers with and without mixed-enzymes and probiotic supplementation 7 d.p.i $(n=8)$. (A) C. jejuni counts in the liver ( $\left.\log _{10} \mathrm{cfu} / \mathrm{g}\right)$; (B) percentage of livers positive for Campylobacter after enrichment. (Red bars represent median values). 
A

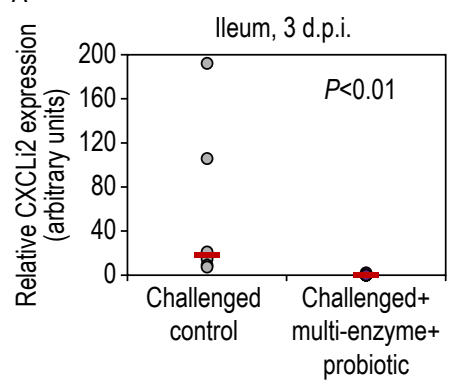

D

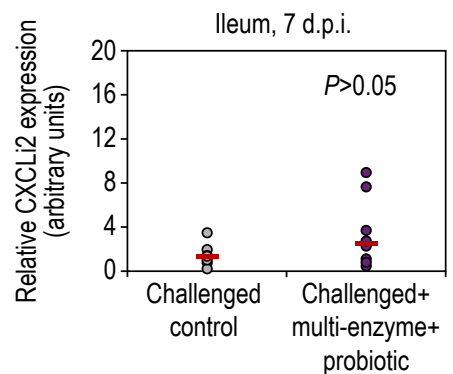

B

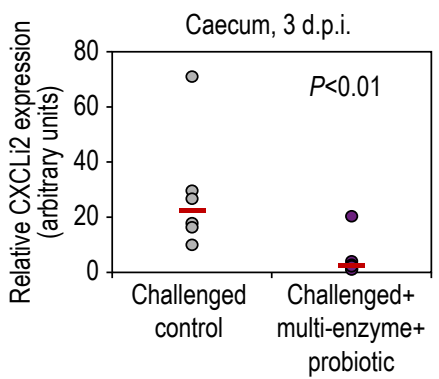

E

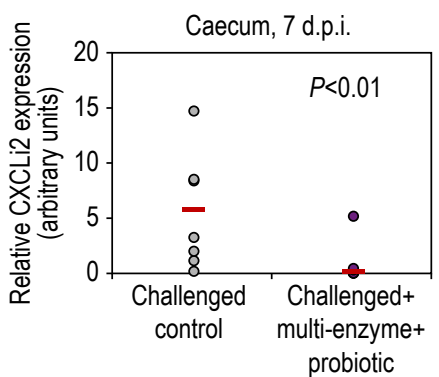

C

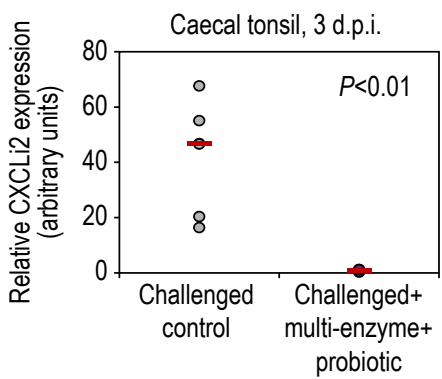

F

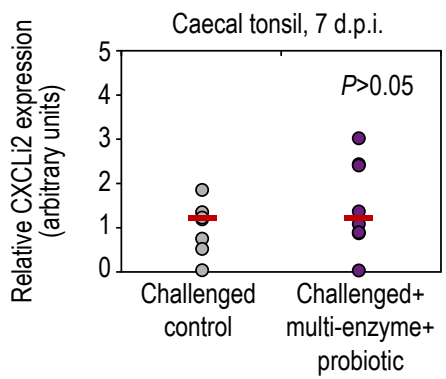

Figure 3. Relative expression of CXCLi2 in the ileum (A) and (D), caecum (B) and (E), and caecal tonsil (C) and (F) of Campylobacter jejuni challenged broilers with and without dietary supplementation of a mixed-enzymes and probiotic combination. Expression levels determined 3 and 7 d.p.i. (Red bars represent median values).

between groups at either time-point in the ileum, whilst in the caecum and caecal tonsils, expression was initially (3 d.p.i.) higher in supplemented birds compared with control birds, but by 7 d.p.i. in the caecum the pattern had reversed, and expression was markedly higher in the control group $(P<0.05$, Table 2).

Expression levels of the regulatory cytokines, IL-10 and TGF- $\beta$, exhibited a broadly similar pattern. At 3 d.p.i., IL10 expression was significantly increased in the caecum and caecal tonsils of MEP supplemented birds compared with unsupplemented challenged control birds $(P<0.01)$. However, at 7 d.p.i. this pattern was reversed and expression in the caecum, caecal tonsils and especially in the ileum was markedly elevated in control birds $(P<0.01 ; P<0.01$ and $P<0.001$ respectively) vs MEP supplemented birds; Table 2 ).

\section{Discussion}

The potential of Campylobacter spp. to cause food-borne disease in humans is clear and undisputed. Its ability to cause adverse clinical or sub-clinical effects in infected poultry is now under debate, due to recent studies linking $C$. jejuni infection with gastrointestinal immune dysregulation, adverse effects on gut physiology, functioning and the microbiome. Such effects can have a negative impact on animal welfare (Humphrey et al., 2014), and are likely to impair nutrition and divert energy resources away from maintenance and growth. The present study sought to address whether and how dietary supplementation with a novel mixed-enzymes and probiotic combination could impact the infection biology of C. jejuni in commercial, fast-growing, broilers with a specific focus on differences in birds' gut immune responses, physiological functioning and microbiome. These factors represent important indicators of the health and functional status of the GIT, which is increasingly being recognised as crucial to achieving optimal nutrition and growth performance in poultry production (Celi et al., 2017). Each bird was considered an experimental unit, since the study was not a nutritional based trial, but rather to generate digesta and tissue samples, as per previous, similar studies regarding microbial and immunological investigations (Tarradas et al., 2020).

The MEP numerically, but substantially, delayed faecal C. jejuni shedding in challenged birds (-98\% vs unsupplemented birds at 3 d.p.i. but was not different at 7 d.p.i.). Supplementation numerically reduced $C$. jejuni colonisation (by 1000-fold vs unsupplemented birds) of the ileal mucosa over the longer term (7 d.p.i.) and inhibited systemic spread of $C$. jejuni to the livers of challenged birds. The additive combination suppressed the initial pro-inflammatory chemokine response seen in the challenged unsupplemented birds and altered the expression of key pro-inflammatory (IL-1 $\beta$, IL-6, IL-17A and IL-17F) and regulatory (IL-10 and TGF- $\beta$ ) cytokines in the gastrointestinal mucosa. These tended to be initially upregulated in ileal and/or caecal tissues of challenged 
Table 2. Relative expression of proinflammatory and regulatory cytokines in gastrointestinal tissue of Campylobacter jejuni challenged ${ }^{1}$ broilers with and without dietary supplementation of a mixed-enzymes and probiotic (MEP) combination. Expression levels determined 3 and 7 d.p.i. (values shown are medians, $n=7$ at 3 d.p.i.; $n=8$ at 7 d.p.i.). ${ }^{2}$

\begin{tabular}{|c|c|c|c|c|c|c|}
\hline & \multicolumn{3}{|l|}{3 days-post-infection } & \multicolumn{3}{|l|}{7 days-post-infection } \\
\hline & Challenged control & Challenged MEP & $P$-value & Challenged control & Challenged MEP & $P$-value \\
\hline \multicolumn{7}{|l|}{ lleal mucosa } \\
\hline IL-1 $\beta$ & 2.36 & 3.53 & 0.886 & 34.34 & 6.51 & 0.016 \\
\hline IL-6 & 0.26 & 3.80 & 0.003 & 5.23 & 0.46 & 0.006 \\
\hline IL-17A & 0.67 & 0.84 & 0.253 & 4.26 & 0.83 & 0.027 \\
\hline IL-17F & 0.78 & 0.71 & 0.943 & 2.68 & 2.37 & 0.753 \\
\hline IL-10 & 0.49 & 1.18 & 0.431 & 12.93 & 0.02 & 0.004 \\
\hline TGF- $\beta$ & 3.16 & 4.15 & 0.345 & 1.99 & 0.56 & 0.001 \\
\hline \multicolumn{7}{|l|}{ Caecum } \\
\hline IL-1 $\beta$ & 1.20 & 9.79 & 0.003 & 0.76 & 0.80 & 0.834 \\
\hline IL-6 & 0.11 & 1.48 & 0.003 & 1.16 & 0.68 & 0.372 \\
\hline IL-17A & 0.07 & 1.41 & 0.003 & 1.71 & 1.02 & 0.345 \\
\hline IL-17F & 0.20 & 1.79 & 0.003 & 14.06 & 0.89 & 0.015 \\
\hline IL-10 & 0.11 & 2.59 & 0.003 & 5.04 & 0.05 & 0.003 \\
\hline TGF- $\beta$ & 0.95 & 1.31 & 0.015 & 1.94 & 0.86 & 0.066 \\
\hline \multicolumn{7}{|c|}{ Caecal tonsil tissue } \\
\hline IL-1 $\beta$ & 0.67 & 3.36 & 0.109 & 1.54 & 0.53 & 0.115 \\
\hline IL-6 & 0.16 & 2.32 & 0.004 & 1.07 & 0.27 & 0.040 \\
\hline IL-17A & 0.32 & 1.76 & 0.006 & 1.19 & 0.54 & 0.345 \\
\hline IL-17F & 0.71 & 1.73 & 0.055 & 1.47 & 0.94 & 0.401 \\
\hline IL-10 & 0.26 & 2.4 & 0.004 & 1.2 & 0.07 & 0.001 \\
\hline TGF- $\beta$ & 0.97 & 2.74 & 0.010 & 1.57 & 0.35 & 0.021 \\
\hline
\end{tabular}

${ }^{1}$ Birds were challenged orally at $\mathrm{d} 21$ with $10^{5} \mathrm{cfu}$ C. jejuni strain M1.

$2 \mathrm{IL}$ - interleukin; TGF = transforming growth factor.

birds receiving MEP, but were generally downregulated by 7 d.p.i., whilst responses in unsupplemented challenged birds tended to follow the opposite pattern.

Modulation of GIT cytokine and chemokine responses by dietary factors, including probiotics, have been demonstrated in previous in vitro and in vivo studies (Gadde et al., 2017; Lee et al., 2010; Paszti-Gere et al., 2012; Waititu et al., 2014). Poultry immune responses to intestinal pathogens such as Eimeria and Salmonella spp., result in an inflammatory response characterised by the upregulation of a variety of pro-inflammatory and anti-inflammatory cytokines, including interferon- $\gamma$, IL-1 $\beta$, IL-2, IL-4, IL-6, IL-17A/F and TGF-beta, IL-10 and IL-13 (Choi and Lillehoj, 2000; Choi et al., 1999; Hong et al., 2006; Kaiser et al., 2000; Kim et al., 2014). These cytokines influence communication between immune cells lining the GIT to orchestrate and regulate the response to pathogen challenge. Studies on C. jejuni are limited, but recent research has identified a defined early pro-inflammatory cytokine response that recruits inflammatory phagocytic cells (heterophils and macrophages) to sites of infection. This leads to alterations in the structure of the intestinal epithelium (Awad et al., 2015) and potential systemic spread to spleen, liver and breast meat (Young et al., 1999).

A key observation of the present study was a substantial spike in the GIT expression of CXCLi2 soon after C. jejuni challenge in unsupplemented challenged birds, which was consistent with an initial proinflammatory response. Smith et al. (2008) and Humphrey et al. (2014) reported similar marked ileal/caecal expression of CXCLi1 and CXCLi2 in the first four days following $C$. jejuni infection among broilers, respectively, with levels generally falling from 7 to 12 d.p.i., as observed in the present study. In birds fed the MEP, the spike in CXCLi2 was notably absent from all gut regions, which suggested a reduced proinflammatory response and influx of immune cells. CXCLi2 has been implicated in the chemoattraction of phagocytic cells, including macrophages (Kaiser and Stäheli 2014; Kogut, 2000), and is a key mediator of the innate immune response. Activated macrophages secrete IL-1 $\beta$, so expression of IL$1 \beta$ may have been expected to rise alongside or following secretion of CXCLi chemokines in the unsupplemented challenged birds. There was some evidence of this in the ileum at 7 d.p.i. but not at 3 d.p.i., whilst in the caecum IL1- $\beta$ expression was less evident in unsupplemented challenged birds than in supplemented challenged birds. Humphrey et al. (2014) and Smith et al. (2008) both reported expression of IL1- $\beta$ immediately following $C$. jejuni infection, which was reduced from 7 to 12 d.p.i.

Expression of IL-6, IL17A and IL17F appeared to be delayed among the challenged control birds when compared with MEP-supplemented birds, as seen in ileal/caecal tissues at 7 d.p.i. with only low expression at 3 d.p.i. The IL-17 cytokine family are pro-inflammatory and recruit neutrophils (equivalent to avian heterophils) and macrophages to sites of infection in mammals (Weaver et al., 2007) and play a key role in the gut in maintaining tight-junction integrity 
and production of antimicrobial peptides, whereas IL-6 has both pro- and anti-inflammatory effects. Recently, Th17 responses have been shown to be critical in the response to $C$. jejuni in chickens and are considered to play a key role in preventing extraintestinal spread (Reid et al., 2016). An upregulation of Th17 cytokines in MEPsupplemented birds may have beneficial consequences for intestinal barrier integrity and reduce systemic spread of bacteria. IL-6 appeared to have an inflammatory role in challenged birds, whereby its delayed appearance in the unsupplemented group may have been due to the effects of $C$. jejuni challenge being unresolved at 7 d.p.i. or to a spike in levels once activated immune cells (e.g. macrophages) were present in abundance. The appearance of marked regulatory IL-10 expression in all gut regions of unsupplemented challenged birds at 7 d.p.i. may have been indicative of an attempt to regulate the inflammatory response. These observations bore some similarities with those of Connerton et al. (2018), who observed increased ileal/caecal expression of IL-6, IL-17A, IL-17F and other proinflammatory cytokines following $C$. jejuni infection that generally did not occur before 9 d.p.i., despite a high level of Campylobacter colonisation occurring as early as 2 d.p.i.

Interestingly, challenged birds fed the additive combination exhibited the opposite pattern of GIT cytokine expression post-infection to that seen in the unsupplemented challenged birds, whereby expression of both pro- (IL$1 \beta$, IL-6, IL-17A, IL-17F) and anti-inflammatory (IL-10, TGF- $\beta$ ) cytokines tended to be higher initially following $C$. jejuni infection in the additive-treated group and then fell to generally low levels by 7 d.p.i. This pattern was broadly compatible with the notion of an earlier enhanced innate and sentinel immune response in MEP-supplemented birds that limited extraintestinal spread, with the avoidance of an unnecessary prolonged inflammatory response in the GIT or a more rapid resolution of challenge in these birds, compared with unsupplemented challenged birds. Early upregulation of IL-10 as a key regulator of cytokine responses may have been indicative of improved control of inflammatory responses in the MEP-treated birds and a reduced likelihood of prolonged inflammation and consequential intestinal pathology. Upregulation of IL-10 by human intestinal cells as a result of TLR signalling has been linked with an increase in epithelial integrity (decreased permeability) that can be induced by commensal bacteria (Hyun et al., 2015). Effects on epithelial barrier integrity were not tested in the present study, but Bacillus spp. probiotics have previously been shown to strengthen the gut barrier of the ileum and caecum in broilers and laying hens challenged with either Eimeria or Campylobacter spp. (Murugesan et al., 2014). This immunomodulatory mechanism of action of probiotics is not fully understood, but is thought to involve an ability to release secondary metabolites with bactericidal and/or immune modulatory effects (Bratz et al., 2015; Latorre et al., 2016; Svetoch et al., 2005). Several in vitro and in vivo studies have variously reported potentially beneficial effects of Bacillus spp. probiotics, including the Syncra ${ }^{\circ}$ AVI product employed in the present study, on gut morphology (villus height and crypt depth), and on the intestinal expression of key pro-inflammatory and anti-inflammatory cytokines in the absence of pathogen challenge (Lee et al., 2010; Waititu et al., 2014; Gadde et al., 2017). The precise nature of effects due to probiotic-based additives on broiler GIT immune responses appears to differ among studies, and the literature considering their effects on immune responses to microbial challenge is currently limited. There is a need for more studies to ascertain precise effects of individual probiotics on the gut immunity alongside the physiological response.

An additional possible consequence of the early IL-17 expression and upregulation of an early anti-inflammatory response to C. jejuni challenge is improved gut epithelial barrier integrity. Effective functioning of the gut barrier is vital to controlling the passage of digesta and microorganisms in and out of the gut lumen, and evidence from human and animal studies suggests that both cytokine secretion and pattern recognition receptors (such as TLRs) play a key role in regulating gut permeability (Capaldo and Nusrat, 2008).

An increase in gut barrier integrity would be expected to reduce the ability of $C$. jejuni to penetrate the GIT mucosa, leading to reduced extraintestinal spread to systemic regions, such as the liver and breast meat. Evidence for reduced GIT C. jejuni colonisation among MEP-supplemented challenged birds in the present study was weak, with only numerical reductions in the colonisation of the ileal mucosa at 7 d.p.i. and where reduced early faecal shedding in MEPsupplemented vs unsupplemented birds was not statistically significant. In a commercial setting, reduced early shedding of $C$. jejuni could reduce the spread of Campylobacter spp. within a flock, since coprophagy of infected faeces is the main route by which birds become infected. In birds naturally colonised by Campylobacter spp., Wealleans et al. (2017) observed a reduction in both Campylobacter and Bacteroides spp. in the caeca of birds supplemented with MEP. They speculated that these effects were likely linked to observed changes in non-starch polysaccharide (NSP) flow through the gut as a result of the fibre-protein hydrolytic activity of the multi-enzyme, which may have reduced substrates for the bacteria in the hindgut. It is clear that $C$. jejuni colonisation can persistently modify caecal microbiota, particularly with regard to reducing beneficial Lactobacillus spp. (Connerton et al., 2018; Kaakoush et al., 2014). The question remains as to what extent probioticenzyme combinations can redress the microbial imbalance in the poultry GIT that may result from C. jejuni infection.

The apparent inhibitory effect of MEP on the spread of $C$. jejuni to the livers of infected birds in the present 
study was marked and, to the authors knowledge, has not previously been reported. Young et al. (1999) demonstrated in vitro that Campylobacter spp. can traverse the intestinal epithelium and recovered it from the spleen and liver of young chicks up to 7 d.p.i. Given the inhibition of extraintestinal spread in the present study was unilateral across birds in the MEP-supplemented group, effects on C. jejuni colonisation and faecal shedding were only weak or not detected, and it seems likely that the mechanism involved may have been improved epithelial barrier integrity rather than a reduction in $C$. jejuni numbers in the GIT per se. For Enterococcus cecorum, a commensal bacteria and opportunistic pathogen that can cause outbreaks of disease, it is thought that the leakage of bacteria across the intestinal epithelial barrier and into the blood circulation (bacterial translocation) is a key step in the pathogenesis of disease and is the route through which the bacteria gain access to distal sites for infection (Wideman, 2016). Setting aside the issue of whether $C$. jejuni intestinal colonisation causes clinical symptoms in the bird, its translocation to edible tissues such as the liver is undesirable from a public health perspective (Moffatt et al., 2016). Therefore, the ability of the MEP to reduce systemic spread of $C$. jejuni could be of interest in the control of $C$. jejuni transmission to humans.

\section{Conclusions}

Overall, the results indicated that MEP supplementation may beneficially modulate the GIT chemokine and cytokine responses, support gut integrity and reduce the systemic spread of $C$. jejuni in broilers, which is an example of nutribiosis, proposed previously by Yitbarek et al. (2019). The implications of such findings warrant further investigation as broiler producers seek to optimise gut health and bird performance.

\section{Conflict of interest}

Authors K. Gibbs, Y. Dersjant-Li and C. Evans are employees of Danisco Animal Nutrition Ltd. The remaining authors declare that the research was conducted in the absence of any commercial or financial relationships that could be construed as a potential conflict of interest.

\section{Acknowledgments}

The authors would like to thank Dr Joelle Buck (Newbury, UK) for her assistance with writing this manuscript, which was sponsored by Danisco Animal Nutrition, The Netherlands, in accordance with Good Publication Practice guidelines.

\section{References}

Awad, W.A., Molnár, J.R., Aschenback, K., Ghareeb, B., Khayal, B., Hess, C., Liebhard, D., Dublecz, K. and Hess, M., 2015. Campylobacter infection in chickens modulates the intestinal epithelial barrier function. Innate Immunity 21: 151-160. https:// doi.org/10.1177/1753425914521648

Bratz, K., Golz, G., Janczy, K., Nöckler, K. and Alter, T., 2015. Analysis of in vitro and in vivo effects of probiotics against Campylobacter spp. Berliner und Münchener Tierärztlliche Wochenschrift 128: 155-162. https://doi.org/10.2376/0005-9366-128-155

Capaldo, C.T. and Nusrat, A., 2008. Cytokine regulation of tight junctions. Biochemica et Biophysica Acta. 1788: 864-871. https:// doi.org/10.1016/j.bbamem.2008.08.027

Celi, P., Cowieson, A.J., Fru-Nji, F., Steinert, R.E., Kleunter, A.-M. and Verlhac, V., 2017. Gastrointestinal functionality in animal nutrition and health: new opportunities for sustainable animal production. Animal Feed Science and Technology 234: 88-100. https://doi. org/10.1016/j.anifeedsci.2017.09.012

Choi, K.D. and Lillehoj, H.S., 2000. Role of chicken IL-2 on gammadelta T-cells and Eimeria acervulina-induced changes in intestinal IL-2 mRNA expression and gammadelta T-cells. Veterinary Immunology and Immunopathology 73: 309-321. https://doi.org/10.1016/s01652427(00)00148-3

Choi, K.D., Lillehoj, H.S. and Zalenga, D.S., 1999. Changes in local IFN-gamma and TGF-beta4 mRNA expression and intraepithelial lymphocytes following Eimeria acervulina infection. Veterinary Immunology and Immunopathology 71: 263-275. https://doi. org/10.1016/s0165-2427(99)00103-8

Connerton, P.L., Richards, P.J., Lafontaine, G.M., O'Kane, P.M., Ghaffar, N., Cummings, N.J., Smith, D.L., Fish, N.M. and Connerton, I.F., 2018. The effect of the timing of exposure to Campylobacter jejuni on the gut microbiome and inflammatory responses of broiler chickens. Microbiome 6: 88. https://doi.org/10.1186/s40168-0180477-5

De Zoete, M.R., Keestra, A.M., Roszczenko, P. and Van Putten, J.P.M., 2010. Activation of human and chicken toll-like receptors by Campylobacter spp. Infection and Immunology 78: 1229-1238. https://doi.org/10.1128/IAI.00897-09

Dersjant-Li, Y., Awati, A., Kromm, C. and Evans, C., 2014. A direct fed microbial containing a combination of 3-strain Bacillus sp. can be used as an alternative to feed antibiotic growth promoters in broiler production. Journal of Applied Animal Nutrition 2: e11, 1-6. https://doi.org/10.1017/jan.2014.4

Dersjant-Li, Y., van de Belt, K., van der Klis, J.D, Kettunen, H., Rinttilä and Awati A., 2015. Effect of multi-enzymes in combination with a direct-fed microbial on performance and welfare parameters in broilers under commercial production settings. Journal of Applied Poultry Research 24: 80-90. https://doi.org/10.3382/japr/pfv003

Dhillon, A.S., Shivaprasad, H.L., Schaberg, D., Wiler, F., Weber S. and Bandli, D., 2006. Campylobacter jejuni infection in broiler chickens. Avian Diseases 50: 55-58. https://doi.org/10.1637/7411-071405R.1 EFSA, 2011. Scientific Opinion on Campylobacter in broiler meat production: control options and performance objectives and/or targets at different stages of the food chain. EFSA Journal 9: 2105. 
EFSA, 2017. The European Union summary report on trends and sources of zoonoses, zoonotic agents and food-borne outbreaks in 2016. EFSA Journal 15: 5077.

FAO, 2016. Probiotics in animal nutrition - production, impact and regulation by Yadav, S., Athol, B., Klieve, V., Dart, P.J. and Bryden, W.L. Edited by Makkar, H.P.S. FAO Animal Production and Health Paper No. 179., FAO, Rome, Italy.

Flores, C., Williams, M., Pieniazek, J., Dersjant-Li, Y., Awati, A., Lee, J.T., 2016. Direct-fed microbial and its combination with xylanase, amylase and protease enzymes in comparison with AGPs on broiler growth performance and foot-pad lesion development. Journal of Applied Poultry Research 25: 328-337. https://doi.org/10.3382/ japr/pfw016

Gadde, U., Oh, S.T., Lee, Y.S., Davis, E., Zimmerman, N. and Rehberger, T., 2017. The effects of direct-fed microbial supplementation, as an alternative to antibiotics, on growth performance, intestinal immune status, and epithelial barrier gene expression in broiler chickens. Probiotics and Antimicrobial Proteins 9: 397-405. https:// doi.org/10.1007/s12602-017-9275-9

Hong, Y.H., Lillehoj, H.S., Lillehoj, E.P. and Lee, S.H., 2006. Changes in immune-related gene expression and intestinal lymphocyte subpopulations following Eimeria maxima infection of chickens. Veterinary Immunology and Immunopathology 114: 259-272. https://doi.org/10.1016/j.vetimm.2006.07.007

Humphrey, S., Chaloner, G., Kemmett, K., Davidson, N., Williams, N., Kipar, A., Humphrey, T. and Wigley, P., 2014. Campylobacter jejuni is not merely a commensal in commercial broiler chickens and affects bird welfare. mBio 5(4): e01364-14. https://doi.org/10.1128/ mBio.01364-14

Hyun, J., Romero, L., Riveron, R., Flores, C., Kanagavelu, S., Chung, K.D., Alonso, A., Sotolongo, J., Ruiz, J., Manukyan, A., Chun, S., Singh, G., Salas, S., Targan, S.R. and Fukata, M., 2015. Human intestinal epithelial cells express IL-10 through Toll-like receptor 4 (TLR4)-mediated epithelial macrophage crosstalk. Journal of Innate Immunity 7: 87-101. https://doi.org/10.1159/000365417

Kaakoush, N.O., Sodhi, N., Chenu, J.W., Cox, J.M., Riordan, S.M. and Mitchell, H.M., 2014. The interplay between Campylobacter and Helicobacter species and other gastrointestinal microbiota of commercial broiler chickens. Gut Pathology 6: 18. https://doi. org/10.1186/1757-4749-6-18

Kaiser, P. and Stäheli, P., 2014. Avian cytokines and chemokines. In: Schat, K.A., Kaspars, P. and Kaiser, P. (eds). Avian immunology. $2^{\text {nd }}$ edition. Academic Press, Cambridge, MA, USA, pp 189-198.

Kaiser, P., Rothwell, L., Galyov, E.E., Barrow, P.A., Burnside, J. and Wigley, P., 2000. Differential cytokine expression in avian cells in response to invasion by Salmonella typhimurium, Salmonella enteritidis and Salmonella gallinarum. Microbiology 146: $3217-$ 3226. https://doi.org/10.1099/00221287-146-12-3217

Kim, W.H., Jeong, J., Park, A.R., Yim, D., Kim, S., Chang, H.H., Yan, S.-H., Kim, D.-H., Lillehoj, H.S. and Min, W., 2014. Downregulation of chicken interleukin-17 receptor A during Eimeria infection. Infection and Immunology 82: 3845-3854. https://doi.org/10.1128/ IAI.02141-14

Kogut, M.H., 2000. Cytokines and prevention of infectious disease in poultry: a review. Avian Pathology 29: 395-404. https://doi. org/10.1080/030794500750047135
La Ragione, R.M. and Woodward, M.J., 2003. Competitive exclusion by Bacillus subtilis spores of Salmonella enterica serotype Enteritidis and Clostridium perfringens in young chickens. Veterinary Microbiology 94: 245-256. https://doi.org/10.1016/ s0378-1135(03)00077-4

Latorre, J.D., Hernandez-Velasco, X., Wolfenden, R.E., Vicente, J.L., Wolfenden, A.D., Menconi, A., Bielke, L.R., Hargis, B.M. and Tellez, G., 2016. Evaluation and selection of Bacillus species based on enzyme production, antimicrobial activity, and biofilm synthesis as direct-fed microbial candidates for poultry. Frontiers in Veterinary Science 3: 95. https://doi.org/10.3389/fvets.2016.00095

Lee, K.W., Lee, S.H., Lillehoj, H.S., Li, G.X., Jang, S.I., Babu, U.S., Park, M.S., Kim, D.K., Lillehoj, E.P., Neumann, A.P., Rehberger, T.G. and Siragusa, G.R., 2010. Effects of direct-fed microbials on growth performance, gut morphometry, and immune characteristics in broiler chickens. Poultry Science 89: 203-216. https://doi. org/10.5713/ajas.2013.13178

Meade, K.G., Narciandi, F., Cahalane, S., Reiman, C., Allan, B. and O'Farrelly, C., 2009. Comparative in vivo infection models yield insights on early host immune response to Campylobacter in chickens. Immunogenetics 61: 101-110. https://doi.org/10.1007/ s00251-008-0346-7

Miles, A.A., Misra, S.S. and Irwin, J.O., 1938. The estimation of the bactericidal power of the blood. Journal of Hygiene 38: 732-49.

Moffatt, C.R.M., Greig, A., Valcanis, M., Gao, W., Seemann, T., Howden, B.P. and Kirk, M.D., 2016. A large outbreak of Campylobacter jejuni infection in a university college caused by chicken liver pate Australia. Epidemiology and Infection 144: 2971-2978. https://doi. org/10.1017/S0950268816001187

Murugesan, G.R., Gabler, N.K. and Persia, M.E., 2014. Effects of directfed microbial supplementation on broiler performance, intestinal nutrient transport and integrity under experimental conditions with increased microbial challenge. British Poultry Science 55: 89-97. https://doi.org/10.1080/00071668.2013.865834

NRC, 1994. Nutrient requirements of poultry. $19^{\text {th }}$ rev. ed. National Academic Press, Washington, DC, USA.

Olukosi, O.A., Beeson, L.A., Englyst, K. and Romero, L.F., 2015 Effects of exogenous proteases without or with NSP-hydrolyzing enzymes on nutrient digestibility and disappearance of non-starch polysaccharides in broiler chickens. Poultry Science 94: 2662-2669. https://doi.org/10.3382/ps/pev260

Park, J.H. and Kim, I.H., 2014. Supplemental effect of probiotic Bacillus subtilis B2A on productivity, organ weight, intestinal Salmonella microflora, and breast meat quality of growing broiler chicks. Poultry Science 93: 2054-2059. https://doi.org/10.3382/ps.201303818

Paszti-Gere, E., Krisztina, S., Csibrik-Nemeth, E., Csizinszky, R., Marosi, A,. Palocz, O., Farkas, O. and Galfi, P., 2012. Metabolites of Lactobacillus plantarum 2142 prevent oxidative stress-induced overexpression of proinflammatory cytokines in IPEC-J2 cell line. Inflammation 35: 1487-1499. https://doi.org/10.1007/s10753-0129462-5 
Reid, W., Close, A., Humphrey, S., Chaloner, G., Lacharme-Lora, L., Rothwell, L., Kaiser, P., Williams, M.J., Humphrey, T.J., Wigley, P. and Rushton, S.P., 2016. Cytokine responses in birds challenged with the human food-borne pathogen Campylobacter jejuni implies a Th17 response. Royal Society of Open Science 3: 150541. https:// doi.org/10.1098/rsos.150541

Romero, L.F., Parson, C.M., Utterback, P.L., Plumstead, P.W. and Ravindran, V., 2013. Comparative effects of dietary carbohydrase enzymes without or with protease on the ileal digestibility of energy and amino acids and AMEn in young broilers. Animal Feed Science and Technology 181: 35-44. https://doi.org/10.1016/j. anifeedsci.2013.02.001

Romero, L.F., Sands, J.S., Indrakumar, S.E., Plumstead, P.W., Dalsgaard, S. and Ravindran, V., 2014. Contribution of protein, starch and fat to the apparent ileal digestible energy of corn- and wheat-based broiler diets in response to exogenous xylanases and amylase without or with protease. Poultry Science 93: 2501-2513. https:// doi.org/10.3382/ps.2013-03789

Shane, S.M., 1992. The significance of Campylobacter jejuni infection in poultry: a review. Avian Pathology 21:189-213. https://doi. org/10.1080/03079459208418836

Shini, S. and Kaiser, P., 2009. Effects of stress, mimicked by administration of corticosterone in drinking water, on the expression of chicken cytokine and chemokine genes in lymphocytes. Stress 12: 388-399. https://doi.org/10.1080/10253890802526894

Smith, C.K, Abuoun, M., Cawthraw, S.A., Humphrey, T.J., Rothwell, L., Kaiser, P., Barrow, P.A. and Jones, M.A., 2008. Campylobacter colonisation of the chicken induces a proinflammatory response in mucosal tissues. FEMS Immunology \& Medical Microbiology 54: 114-121. https://doi.org/10.1111/j.1574-695X.2008.00458.x

Smith, C.K., Kaiser, P., Rothwell, L., Humphrey, T., Barrow, P.A. and Jones, M.A., 2005. Campylobacter jejuni-induced cytokine responses in avian cells. Infection and Immunity 73: 2094-2100. https://doi. org/10.1128/IAI.73.4.2094-2100.2005

Stanley, D., Hughes, R.J., Geier, M.S. and Moore, R.J., 2016. Bacteria within the gastrointestinal tract microbiota correlated with improved growth and feed conversion: challenges presented for the identification of performance enhancing probiotic bacteria. Frontiers in Microbiology 7: 187. https://doi/org/10.3389/fmicb.2016.00187
Svetoch, E.A., Stern, N.J., Eruslanov, B.V., Kovalev, Y.N., Volodina, L.I. and Perelygin, V.V., 2005. Isolation of Bacillus circulans and Paenibacillus polymyxa strains inhibitory to Campylobacter jejuni and characterisation of associated bacteriocins. Journal of Food Protection 68: 11-27. https://doi.org/10.4315/0362-028x-68.1.11

Tarradas, J., Tous, N., Esteve-Garcia, E. and Brufau, J., 2020. The control of intestinal inflammation: a major objective in the research of probiotic strains as alternatives to antibiotic growth promoters in poultry. Microorganisms 8: 158. https://doi.org/10.3390/ microorganisms 8020148

Waititu, S.M., Yitbarek, A., Matini, E., Echeverry, H., Kiarie, E., Rodriguez-Lecompte, J.C. and Nyachoti, C.M., 2014. Effect of supplementing direct-fed microbials on broiler performance, nutrient digestibilities, and immune responses. Poultry Science 93: 625-635. https://doi.org/10.3382/ps.2013-03575

Wealleans, A.L., Walsh, M.C., Romero, L.F. and Ravindran, V., 2017. Comparative effects of two multi-enzyme combinations and a Bacillus probiotic on growth performance, digestibility of energy and nutrients, disappearance of non-starch polysaccharides and gut microflora in broiler chickens. Poultry Science 96: 4287-4297. https://doi.org/10.3382/ps/pex226

Weaver, C.T., Hatton, R.D., Mangan, P.R., Harrington, L.E., 2007. IL17 family cytokines and the expanding diversity of effector $\mathrm{T}$ cell lineages. Annual Review of Immunology 25: 821-852. https://doi. org/10.1146/annurev.immunol.25.022106.141557

Wideman, R.F., 2016. Bacterial chondronecrosis with osteomyelitis and lameness in broilers: a review. Poultry Science 95: 325-344. https://doi.org/10.3382/ps/pev320

Yitbarek, A., Gibbs, K., Evans, C. and Overby, D., 2019. Direct fed microbials in the era of antibiotic free production - leveraging nutribiosis to illuminate the effect on gut microbiota and the immune system. Proceedings 2019 Minnesota Nutrition Conference. MN, USA. Available at: https://mnnutritionconf.umn.edu/summaryyitbarek.

Young, C.R., Ziprin, R.L., Hume, M.E. and Stanker, L.H., 1999. Dose response and organ invasion of day-of-hatch leghorn chicks by different isolates of Campylobacter jejuni. Avian Diseases 43: 763767. 
